\title{
CATARACT SURGERY IN AFRICAN-AMERICANS WITH LONG ANTERIOR LENS ZONULES
}

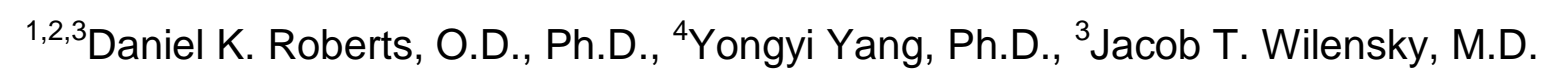

${ }^{1}$ Illinois Eye Institute, Department of Clinical Education, Illinois College of Optometry, Chicago, IL

${ }^{2}$ University of Illinois at Chicago, Department of Epidemiology and Biostatistics, Chicago, IL

${ }^{3}$ University of Illinois at Chicago, Department of Ophthalmology and Visual Sciences, Chicago, IL

${ }^{4}$ Illinois Institute of Technology, Department of Electrical and Computer Engineering, Chicago, IL

Submitted to: Clinical and Experimental Ophthalmology

Corresponding Author:

Daniel K. Roberts

Illinois Eye Institute

3241 S. Michigan Avenue

Chicago, Illinois 60616

Phone: $312-949-7318$

Fax: 312-949-7379

E-mail: droberts@ico.edu

Acknowledgements

Presented at Annual Meeting of the American Ophthalmological Society, Charleston, SC, U.S.A., May, 2012.

Institutional review board approval was obtained for the work related to this letter.

Support: NEI Grant K23 EY0181883 (DKR).

Conflict of interest: None 
Long anterior zonules (LAZ) are characterized by the presence of zonular fibers, central to the normal insertion zone on the anterior lens capsule. ${ }^{(1)}$ They sometimes result in a markedly reduced zonule-free zone (Fig. 1), and have received attention relative to cataract extraction because of concern that their disruption during capsulectomy may lead to capsular tearing and intraocular lens instability. ${ }^{(2 ; 3)}$ So far though, there has been minimal report on surgical outcomes in eyes with LAZ.

Due to involvement in separate study of $L A Z,{ }^{(4)}$ we receive questions whether these subjects experience complications following cataract extraction. Thus, we retrospectively reviewed the outcomes of people having cataract surgery who were identified from a dataset of 227 LAZ subjects from an urban, eye care teaching facility in Chicago, IL, USA. The 227 members had been identified during primary eye care over $12-13$ years, and were included because they had zonular fibers $\geq 1.0 \mathrm{~mm}$ central to the normal, rather well-demarcated, anterior zonule termination zone which is about $2.5 \mathrm{~mm}$ anterior to equator $^{(3)}$ (Fig. 1). Racial composition of this LAZ dataset reflected the predominantly African-American clinic population from which it was derived. All subjects had LAZ confirmed on one or more occasions during dilated eye examination.

Upon review of current records of people in the dataset, we found 19 (16 females, 29 eyes) who had cataract surgery at some point after their initial LAZ detection (Table 1). Although quantification of $L A Z$, in terms of clock-hour, number, and anterior extent wasn't possible, notes and photographs helped estimate that 27 of 29 eyes had "moderate" or "heavy" LAZ based the following criteria: Mild=1-9 countable LAZ; Moderate $=10-49 L A Z$; Heavy $=50$ or more $L A Z$. 
Circumstances after initial LAZ detection, and leading up to surgery, were variable. Typically, LAZ were detected during regular eye care, and subjects were seen afterwards at varied intervals, often by multiple doctors. When surgery was needed, there wasn't a consistent surgical referral pattern, as consultation was requested by different practitioners, with some subjects even seeking cataract care by independent specialists on their own. As summarized in Table 1, we did not find evidence of intraocular lens dislocation/decentration, nor other trends suggestive of untoward surgical outcomes, among the operated eyes. The median subject age at LAZ detection was 72.1 years (62.6 to 83.9 years), and at surgery for right eyes was 75.3 years (66.8 to 83.4 years) and 75.0 (65.5 to 87.1 years) for left eyes. Median time after surgery for right eyes was 1.7 years ( 0.4 to 5.6 years) and 2.3 years ( 0.1 to 6.8 years) for left eyes. Based on available information, 15 eyes were operated in one of three different academic medical centers, 13 eyes in private practice settings, and 1 in an unknown location. Specific surgeon(s) couldn't always be determined, but we estimated that procedures were performed by at least 12 different physicians. Surgical records were often not obtainable.

Although we did not find evidence of complications, we are not suggesting that this rules out any elevated risk for capsular tears in eyes with LAZ. The detailed nature of this relationship remains unknown and may be complex. Also, the findings from our sample aren't necessarily generalizable. Nonetheless, the fact that these patients did well in the hands of multiple surgeons in different settings, may be informative. Although this retrospective evaluation may be advantageous because there weren't planned protocols that might bias surgical efforts toward greater caution, it also 
prevented knowing other information, including decisions that might have been made by surgeons to exercise greater caution. Also, we could not determine what level of awareness of LAZ presence the various surgeons had at surgery. Although subjects were part of the LAZ dataset, notation of LAZ presence wasn't carried through all clinical records. Among the few surgical notes available, it was interesting that LAZ weren't necessarily mentioned. Based on our experience with $L A Z$, and how difficult the trait can be to discern, we suspect it's common for them to go undetected at the time of surgery, especially if special staining techniques are not employed. ${ }^{(2)}$

Although capsulectomy involving the normal zonular insertion zone may promote radial tearing ${ }^{(5)}$ the effect of LAZ disruption is unclear because of certain peculiarities. For example, although one might expect to "visually trace" LAZ fibers from their central tip all the way to their concealment behind the pupil border, they often seem to abruptly "terminate" within the mid-periphery of the anterior capsule (Fig. 1). Whether this type of "segmental LAZ " has extension that is "concealed" deeper in the capsule is unknown, and it is unclear how this might influence risk of capsular tears. Also, unlike normal zonules at their anterior insertion, LAZ fibers often have considerable space between individual fibers, they may be few in number, they may be thicker, and they may be variable in length even within the same eye. ${ }^{(4)}$ 


\section{Reference List}

(1) Sturrock GD, Tripathi RC. Pigmented lens striae. Br J Ophthalmol 1976; 60(4):287293.

(2) Kelty PJ, Snyder ME, Schneider S. Indocyanine green staining of anteriorly placed zonules. J Cataract Refract Surg 2003; 29(11):2229-2231.

(3) Lim SJ, Kang SJ, Kim HB, Kurata Y, Sakabe I, Apple DJ. Analysis of zonular-free zone and lens size in relation to axial length of eye with age. J Cataract Refract Surg 1998; 24(3):390-396.

(4) Roberts DK, Ayyagari R, McCarthy B, Xie H, Davis F, Wilensky JT. Investigating Ocular Dimensions in African Americans With Long Anterior Zonules. J Glaucoma 2013; 22(5):393-397.

(5) Assia El, Apple DJ, Tsai JC, Morgan RC. Mechanism of radial tear formation and extension after anterior capsulectomy. Ophthalmology 1991; 98(4):432-437. 


\section{Figure Legend}

Figure 1. Retroillumination example of mostly non-pigmented LAZ (top left). Digital enhancement image showing normal zonular insertion zone (arrows) (top right). Enhanced photo of 360 degree LAZ causing a small zonule-free zone (ZFZ) (bottom left). Pigmented LAZ (arrow) on anterior capsule following cataract extraction (bottom middle, Subject \#11). Enhanced photo of "segmental LAZ" (arrows) (bottom right). 